\title{
A Multiplexed HILIC-MS/HRMS Assay for the Assessment of Transporter Inhibition Biomarkers in Phase I Clinical Trials: Isobutyryl-Carnitine as an Organic Cation Transporter (OCT1) Biomarker
}

Lina Luo*1, Ragu Ramanathan ${ }^{1}$, Lauren Horlbogen ${ }^{1}$, Sumathy Mathialagan ${ }^{2}$, Chester Costales ${ }^{2}$, Manoli Vourvahis $^{3}$, Christopher L. Holliman ${ }^{1}$, and A. David Rodrigues ${ }^{2}$

\begin{abstract}
${ }^{1}$ Bioanalytical Group, Medicine Design, Pfizer Worldwide Research \& Development, Pfizer Inc., 445 Eastern Point Road, Groton, CT 06340, USA

${ }^{2}$ Transporter Sciences Group, Medicine Design, Pfizer Worldwide Research \& Development, Pfizer Inc., 445 Eastern Point Road, Groton, CT 06340, USA

${ }^{3}$ Clinical Pharmacology, Pfizer Global Product Development, Pfizer Inc., 235 East 42nd Street, New York City, NY, 10017, USA
\end{abstract}

* To whom correspondence should be addressed:
E-mail: lina.luo@pfizer.com Tel: 860.686.2270

This supporting information document contains:

Method development on the chromatographic separation of isobutyryl-carnitine and butyryl-carnitine and the rational to use the HILIC method

Figure S1: Calibration curves demonstrating parallelism of endogenous analytes in surrogate and authentic matrices.

Figure S2: Chromatograms of Blanks and LLOQ for each analyte.

Figure S3: Endogenous compounds with significant changes in the FIH study after PFE-1 administration.

Figure S4: Potential biomarker changes in plasma with metformin PFE-2 DDI study

Table S1: PFE-1 and PFE-2 as In Vitro Inhibitors of Various Human Drug Transporters and Agency Drug-Drug Interaction Risk Assessment

Table S2: Analytical Characteristics of the UHPLC-MS/HRMS Method

Table S3: Slope comparison demonstrating parallelism in authentic and surrogate matrices

Table S4: Intra- and Inter-day Assay Precision of the Endogenous Analytes in Human Control Plasma

Table S5: Matrix Effect for the Six Analytes in Human Plasma

Table S6: Recovery of the Six Analytes in Human Plasma 


\section{Method development on the chromatographic separation of isobutyryl-carnitine and butyryl-carnitine, and the rational to use the HILIC method.}

According to literature reports, isobutyryl-carnitine (IBC) and butyryl-carnitine (BC) are hard to chromatographically separate by HILIC, reverse phase, or even chiral columns. To separate these two isomers by the UHPLC system, various columns and UHPLC conditions were tested. The following results show the best separation we achieved on an Acquity BEH C18 column, $2.1 \times 150 \mathrm{~mm}, 1.7 \mu \mathrm{M}$, following the same sample extraction procedure as the HILIC method described in the manuscript, except the final extract composition was $75 \%$ aqueous vs $10 \%$ aqueous in the HILIC method.

Flow rate: $0.3 \mathrm{~mL} / \mathrm{min}$

Mobile phase A: $20 \mathrm{mM}$ ammonium formate with $0.1 \%$ Formic Acid in $\mathrm{H} 2 \mathrm{O}$

Mobile phase B: Methanol

Run Time: 15 min

1. Examples of IBC and BC standards in surrogate matrix on the C18 column, IBC eluted at $1.8 \mathrm{~min}$ followed by $\mathrm{BC}$ at $1.9 \mathrm{~min}$ from low to high standards; $\mathrm{d} 3-\mathrm{IBC}$ also eluted at $1.8 \mathrm{~min}$.
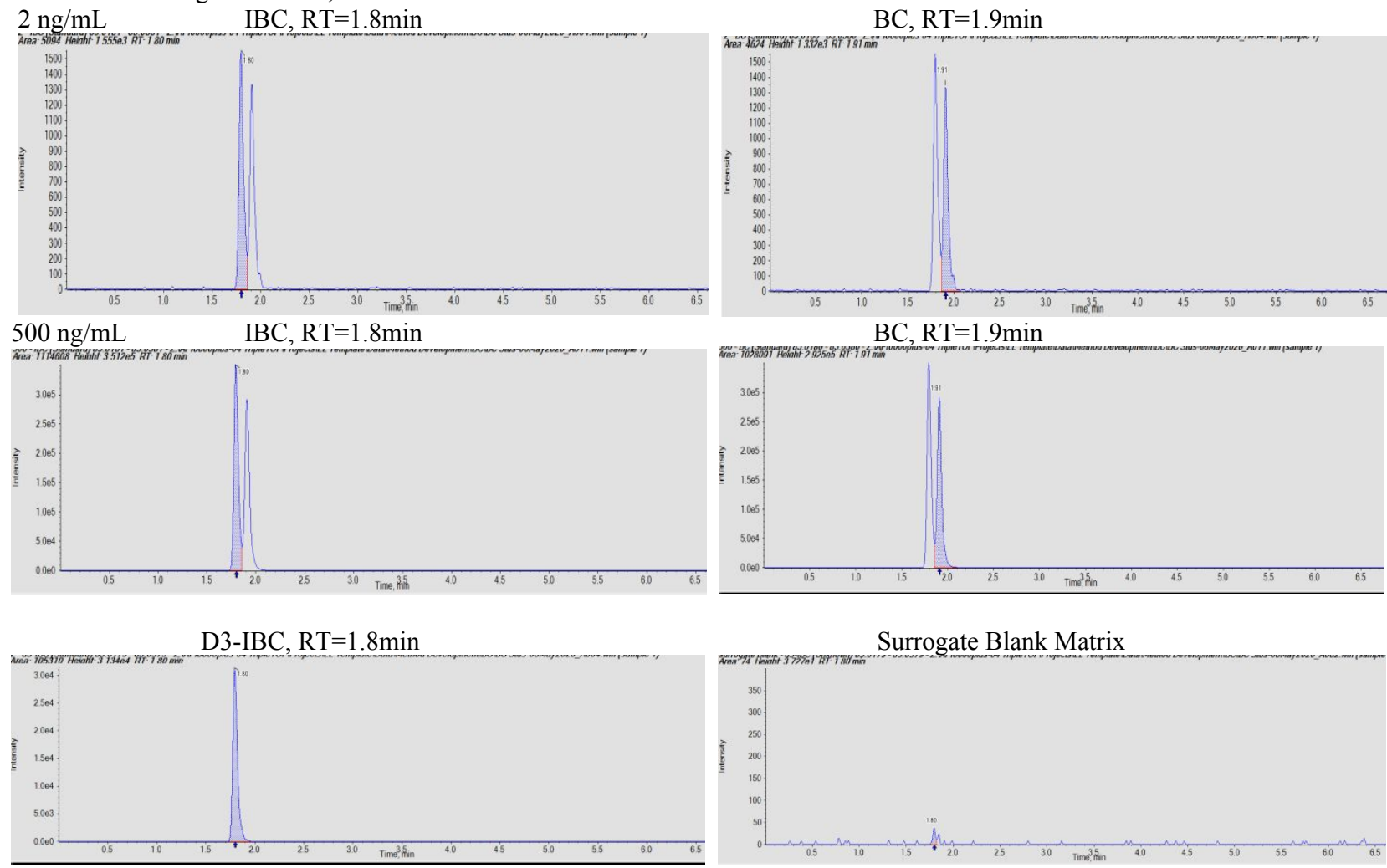

2. Linearity of BC \& IBC on RPHPLC-MS/HRMS, $1-500 \mathrm{ng} / \mathrm{mL}$ IBC, $\mathrm{R}=0.9989$

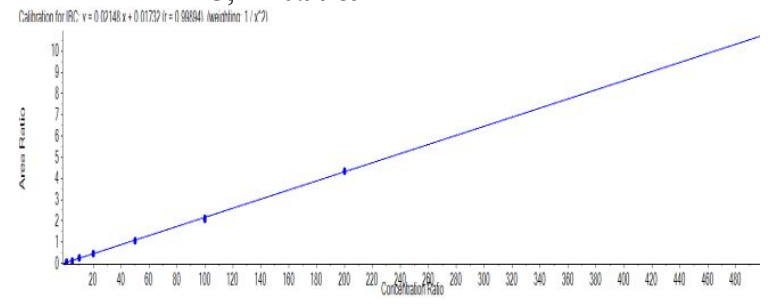

$\mathrm{BC}, \mathrm{R}=0.9949$

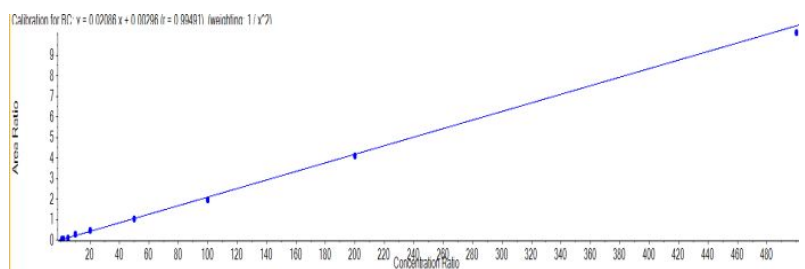

3. Example of BC and IBC chromatograms from the human study plasma samples using the above RPHPLC-MS/HRMS method. A single peak of IBC with $\mathrm{RT}=1.8 \mathrm{~min}$ was observed in most of the human plasma samples (below left), except for the occasional observation of a minor shoulder peak at the retention time of $\mathrm{BC}$ (below right)
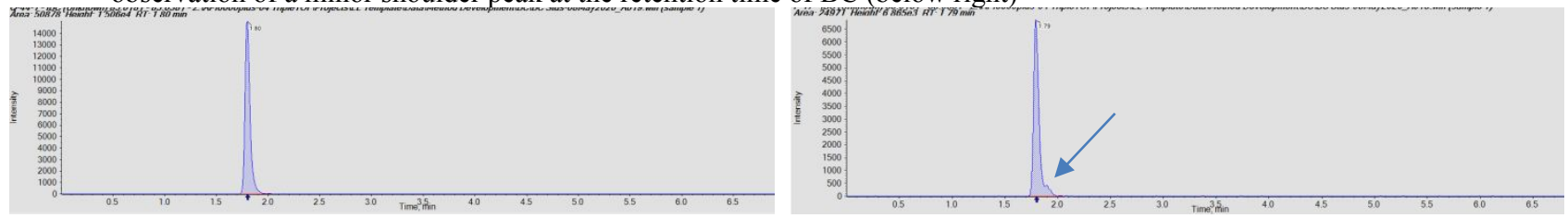
Furthermore, we compared the BC and IBC Response factors using the HILIC-MS/HRMS Method.

$\mathrm{BC} 10 \mathrm{ng} / \mathrm{mL}$ in neat solution (ACN: $\mathrm{H} 2 \mathrm{O}, 50 / 50)$

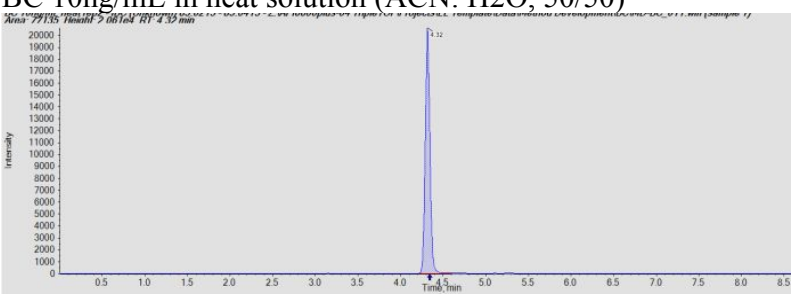

IBC $10 \mathrm{ng} / \mathrm{mL}$ in neat solution $(\mathrm{ACN}: \mathrm{H} 2 \mathrm{O}, 50 / 50)$

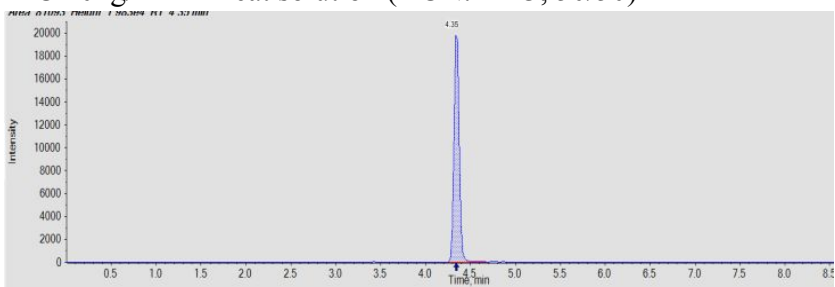

$\mathrm{BC}$ and IBC average response difference in neat solution: Diff $\%=0.71 \%$

$\mathrm{BC} 10 \mathrm{ng} / \mathrm{mL}$ spiked into authentic plasma lot\#1

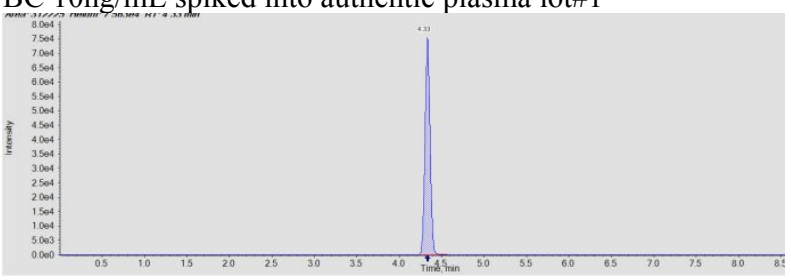

IBC $10 \mathrm{ng} / \mathrm{mL}$ spiked into authentic plasma lot\#1

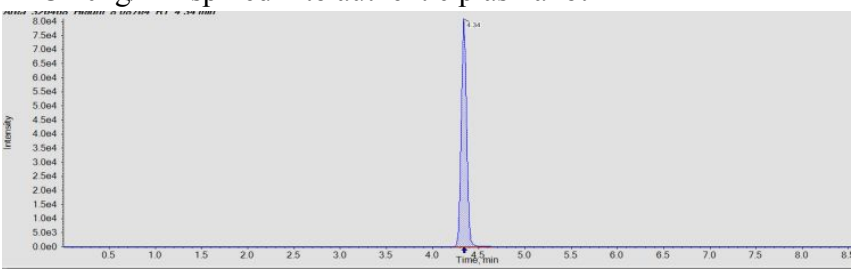

$\mathrm{BC}$ and IBC average response difference in authentic matrix: Diff $\%=4.4 \%$

The data demonstrates that BC and IBC have same response on the system with the HILIC method giving confidence that the small amounts of $\mathrm{BC}$ in the samples will not disproportionately contribute to the IBC quantitation due to differences in isomer response factors. Based on the experimental results, we concluded there is no need to use the reverse phase separation with the longer column $(150 \mathrm{~mm})$ and run time (15min) for the separation of BC and IBC.

We further calculated the relative percent differences of the IBC concentrations measured using the HILIC and reverse phase chromatography. Representative examples from the clinical studies are shown below. There is a high bias towards the reverse phase method but we note that the reverse phase concentrations were generated during method development and the method was not characterized as rigorously as the HILIC method.

\begin{tabular}{|c|c|}
\hline Sample ID & Relative Diff $\%$ \\
\hline F-41 & 14.5 \\
\hline F-41-D10 & 18.2 \\
\hline F-42 & 15.2 \\
\hline F-43 & 11.5 \\
\hline F-47 & 14.8 \\
\hline Q-44-1 & 17.9 \\
\hline Q-44-10 & 17.1 \\
\hline Q-233-1 & 3.9 \\
\hline Q-233-10 & 11.6 \\
\hline
\end{tabular}

No significant difference of the measurements (within $\pm 20 \%$ of bioanalytical error) was observed between the two methods since very little $\mathrm{BC}$ is present in the plasma samples. Therefore, the HILIC methodology is deemed appropriate and sufficient to use here even though the isobaric compounds of $\mathrm{BC}$ and IBC are indistinguishable on the HILIC column. 


\section{A) Creatinine (20-20000 $\mathrm{ng} / \mathrm{mL})$}

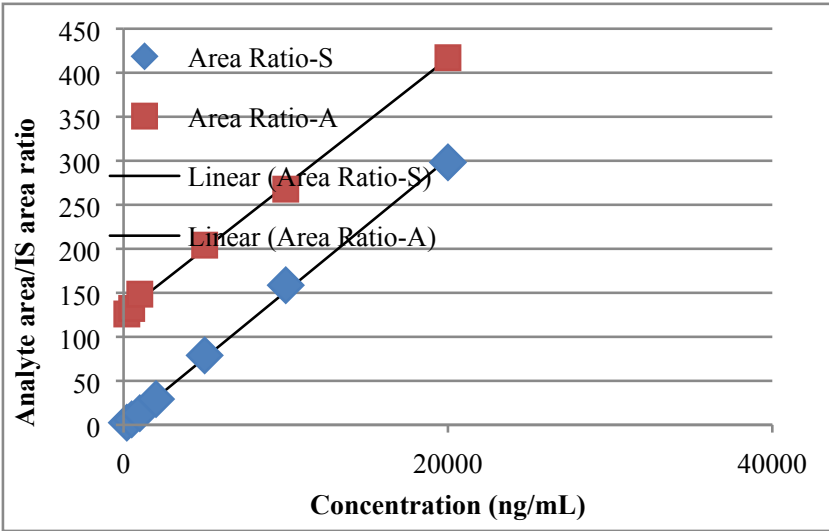

C) Thiamine $(0.5-100 \mathrm{ng} / \mathrm{mL})$

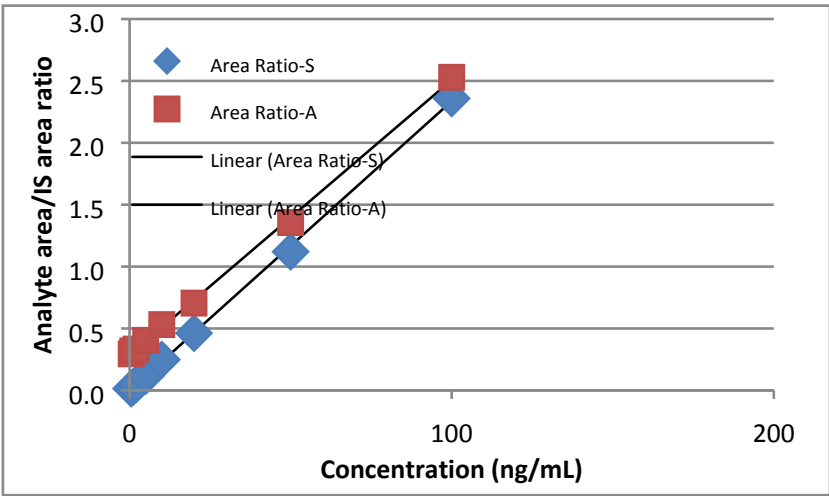

B) 1-NMN $(1-1000 \mathrm{ng} / \mathrm{mL})$

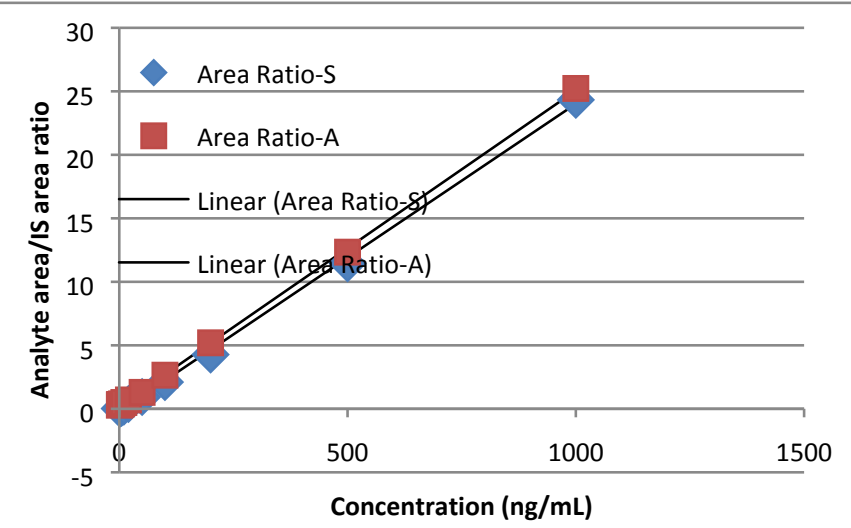

D) Carnitine $(50-20000 \mathrm{ng} / \mathrm{mL})$

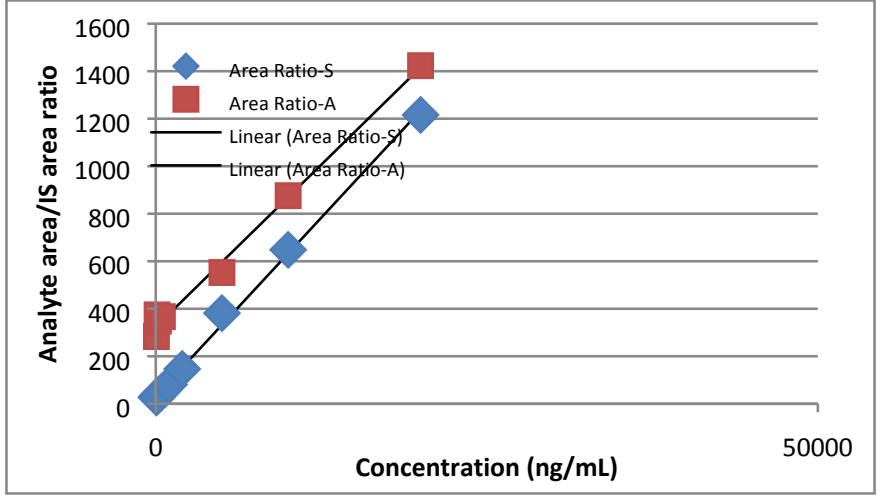

Figure S1. Calibration curves demonstrating parallelism of endogenous analytes in surrogate and authentic matrices. A: Authentic; S: Surrogate 
A). 1-NMN: 1-1000ng/mL
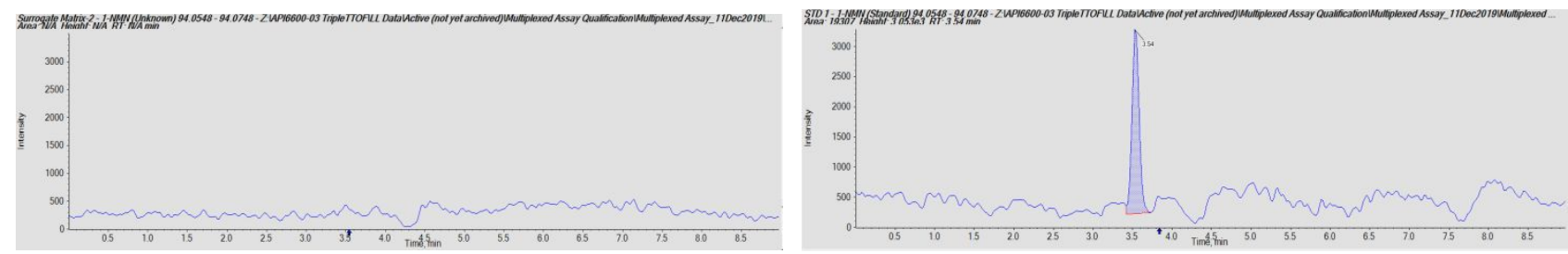

B). IBC: $0.5-500 \mathrm{ng} / \mathrm{mL}$
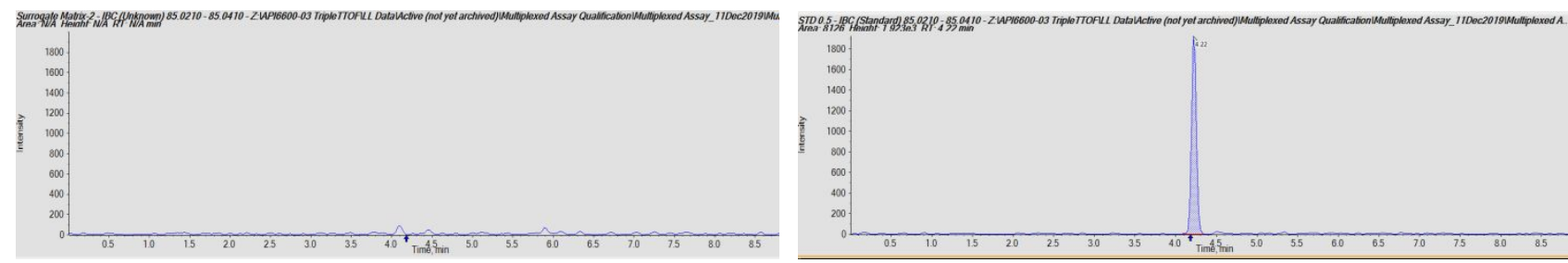

C). Creatinine: $20-20,000 \mathrm{ng} / \mathrm{mL}$
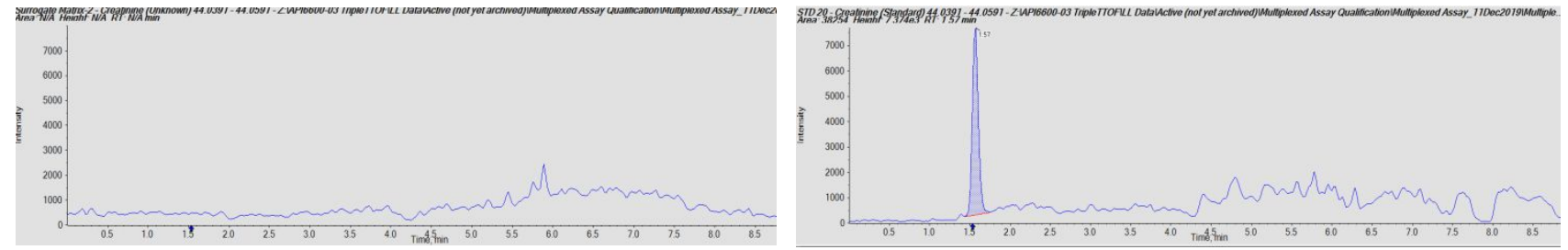

D). Thiamine: $0.5-100 \mathrm{ng} / \mathrm{mL}$
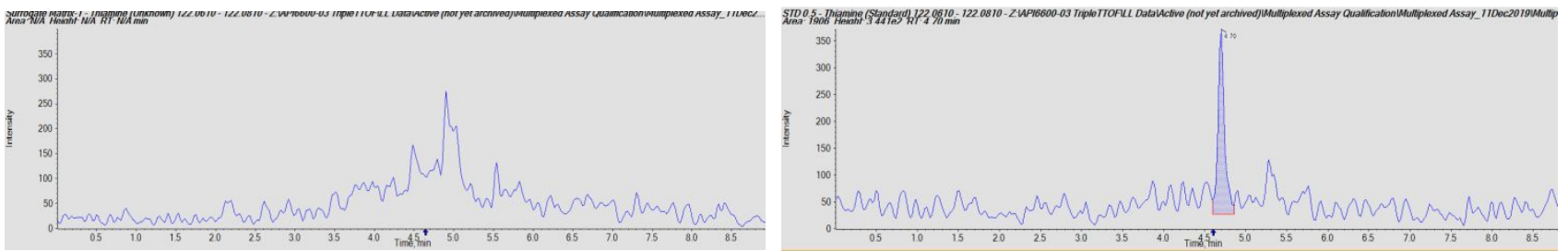

E). Carnitine: $50-20,000 \mathrm{ng} / \mathrm{mL}$
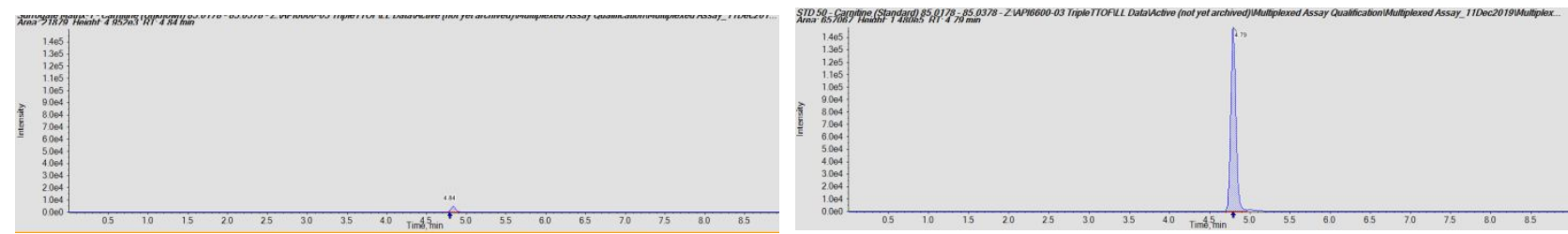

\section{F). Metformin: 0.5-20,000ng/mL}
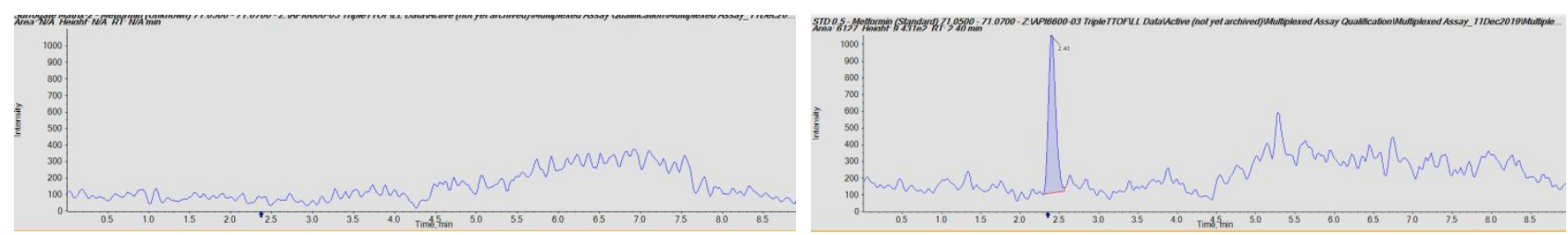

Figure S2. Chromatograms of Blanks and LLOQ for each analyte 
1-NMN Concentrations in Pooled Plasma

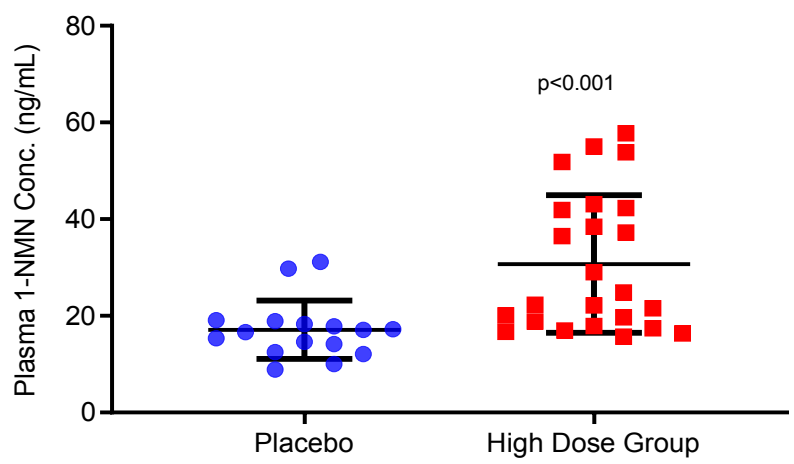

Overall Carnitine Comparison in Pooled Plasma

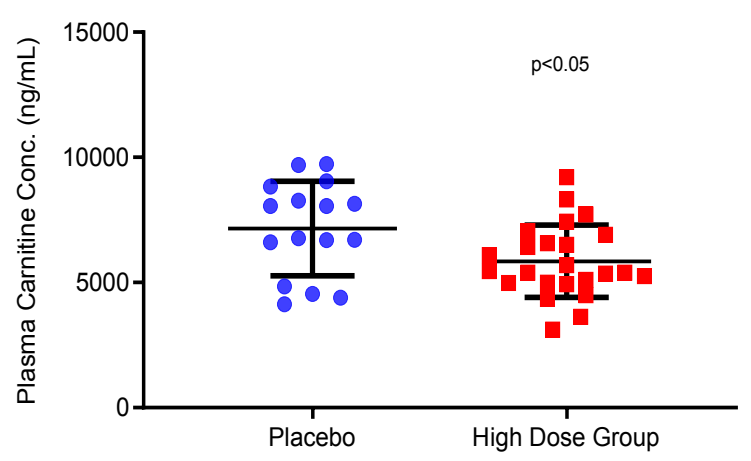

Figure S3. Endogenous compounds with significant changes in the FIH study with PFE-1 administration. 
1-NMN Concentration Over Time

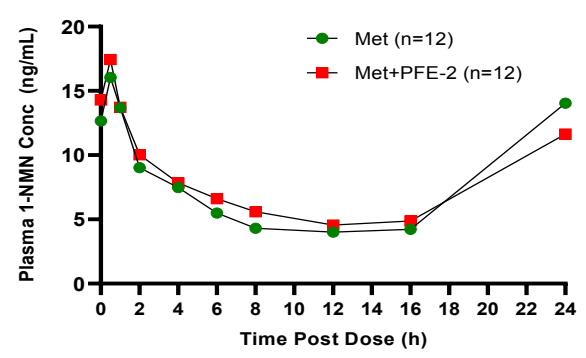

147

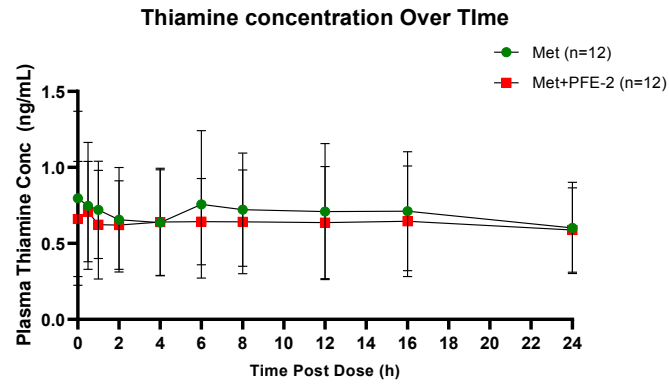

Carnitine Conc. Comparison

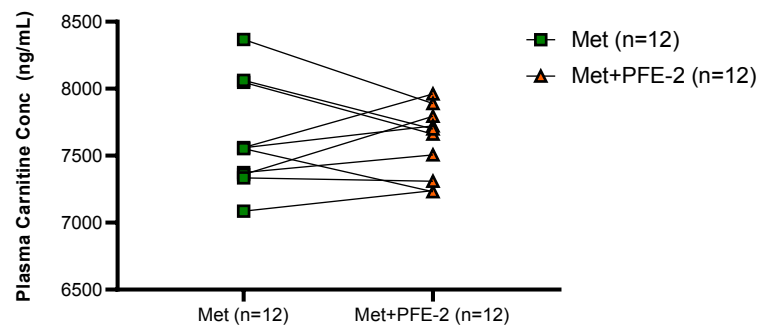

Metformin Conc. Comparison

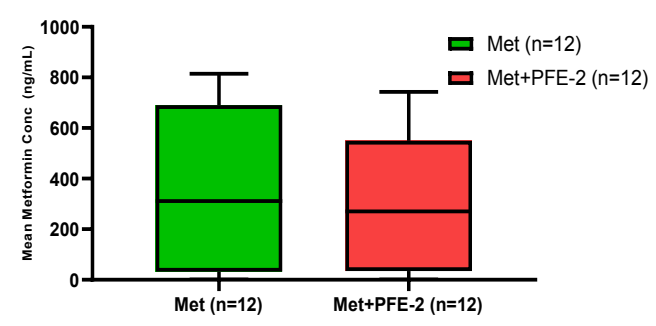

Figure S4. Potential biomarker changes in plasma with metformin PFE-2 DDI study. 
Table S1. PFE-1 and PFE-2 as In Vitro Inhibitors of Various Human Drug Transporters and Agency Drug-Drug Interaction Risk Assessment.

\begin{tabular}{|c|c|c|c|c|}
\hline \multirow[b]{2}{*}{ Parameter ${ }^{\mathrm{a}}$} & \multicolumn{2}{|c|}{ Organic Cation Transporter } & \multicolumn{2}{|c|}{ Multidrug \& Toxin Extrusion Protein } \\
\hline & $\begin{array}{l}\text { OCT1 } \\
\text { (Hepatic) }\end{array}$ & $\begin{array}{l}\text { OCT2 } \\
\text { (Renal) }\end{array}$ & $\begin{array}{l}\text { MATE1 } \\
\text { (Renal/Hepatic) }\end{array}$ & $\begin{array}{l}\text { MATE2K } \\
\text { (Renal) }\end{array}$ \\
\hline \multicolumn{5}{|l|}{ PFE-1 ${ }^{b}$} \\
\hline $\mathrm{IC}_{50}(\mu \mathrm{M})$ & $0.38 \pm 0.04$ & $0.84 \pm 0.03$ & $5.3 \pm 0.25$ & $14.7 \pm 0.84$ \\
\hline $\begin{array}{l}\mathrm{C}_{\max , \mathrm{u}} / \mathrm{IC} \mathrm{C}_{50} \\
\text { (Risk Threshold) }\end{array}$ & - & $\begin{array}{l}1.3 \\
(\geq 0.1)\end{array}$ & $\begin{array}{l}0.21 \\
(\geq 0.02)\end{array}$ & $\begin{array}{l}0.07 \\
(\geq 0.02)\end{array}$ \\
\hline $\begin{array}{l}\text { R-value } \\
\text { (Risk Threshold) }\end{array}$ & $\begin{array}{l}15.7 \\
(\geq 1.1)\end{array}$ & - & - & - \\
\hline \% Inhibition & 94 & 57 & 17 & 7 \\
\hline \multicolumn{5}{|l|}{ PFE-2 ${ }^{c}$} \\
\hline $\mathrm{IC}_{50}(\mu \mathrm{M})$ & 44.2 & $>300$ & 5.5 & 10.7 \\
\hline $\begin{array}{l}\mathrm{C}_{\max , \mathrm{u}} / \mathrm{IC}_{50} \\
\text { (Risk Threshold) }\end{array}$ & - & $\begin{array}{l}0.004 \\
(\geq 0.1)\end{array}$ & $\begin{array}{l}0.24 \\
(\geq 0.02)\end{array}$ & $\begin{array}{l}0.13 \\
(\geq 0.02)\end{array}$ \\
\hline $\begin{array}{l}\text { R-value } \\
\text { (Risk Threshold) }\end{array}$ & $\begin{array}{l}1.4 \\
(\geq 1.1)\end{array}$ & - & - & - \\
\hline$\%$ Inhibition & 29 & 0 & 20 & 11 \\
\hline
\end{tabular}

${ }^{\mathrm{a}} \mathrm{IC}_{50}$, concentration of PFE-1 and PFE-2 rendering 50\% inhibition of transporter activity in vitro; metformin used as substrate for organic cation transporter 1 (OCT1) and 2 (OCT2), as well as multidrug and toxin extrusion protein 1 (MATE1) and 2K (MATE2K). The $\mathrm{IC}_{50}$ is related to a measure of exposure and applied to regulatory agency cutoff to determine the risk of a drugdrug interaction.

$\%$ inhibition was calculated as;

$$
\% \text { Inhibition }=\frac{[I]}{[I]+I C_{50}} \times 100
$$

For OCT2, MATE1 and MATE2K, $[\mathrm{I}]=\mathrm{C}_{\max , \mathrm{u}}$. For OCT2, $[\mathrm{I}]=\mathrm{I}_{\mathrm{in}, \max , \mathrm{u}}$.

${ }^{b}$ For PFE-1, dose $(\mathrm{mg})=100$; molecular weight $=389.41$; fraction unbound in plasma $\left(\mathrm{f}_{\mathrm{u}, \mathrm{p}}\right)=0.56$; maximal total concentration in plasma $\left(\mathrm{C}_{\text {max,tot }}\right)=2 \mu \mathrm{M}$; maximal unbound concentration in plasma $\left(\mathrm{C}_{\text {max }, \mathrm{u}}\right)=1.1 \mu \mathrm{M}$; blood-to-plasma ratio in vitro $\left(\mathrm{R}_{\mathrm{B}}\right)=1.2$; fraction absorbed $\left(\mathrm{f}_{\mathrm{a}}\right)=1$; fraction surviving gut first pass $\left(\mathrm{f}_{\mathrm{g}}\right)=1$; absorption rate constant $\left(k_{\mathrm{a}}\right)=0.1$ min $^{-1}$; hepatic blood flow $\left(\mathrm{Q}_{\mathrm{h}}\right)$ $=1.5 \mathrm{~L} / \mathrm{min}$; estimated maximal total portal concentration $\left(\mathrm{I}_{\mathrm{in}, \max }\right)=10 \mu \mathrm{M}$; estimated maximal unbound portal concentration $\left(\mathrm{I}_{\mathrm{in}, \max , \mathrm{u}}\right)=5.6 \mu \mathrm{M}$.

${ }^{c}$ For PFE-2, dose $(\mathrm{mg})=200$; molecular weight $=323.42$; fraction unbound in plasma $\left(f_{u, p}\right)=0.46$; maximal total concentration in plasma $\left(\mathrm{C}_{\text {max,tot }}\right)=2.91 \mu \mathrm{M}$; maximal unbound concentration in plasma $\left(\mathrm{C}_{\text {max }, \mathrm{u}}\right)=1.34 \mu \mathrm{M}$; blood-to-plasma ratio in vitro $\left(\mathrm{R}_{\mathrm{B}}\right)=$ 1.15 ; fraction absorbed $\left(\mathrm{f}_{\mathrm{a}}\right)=1$; fraction surviving gut first pass $\left(\mathrm{f}_{\mathrm{g}}\right)=1$; absorption rate constant $\left(k_{\mathrm{a}}\right)=0.1$ min $^{-1}$; hepatic blood flow $\left(\mathrm{Q}_{\mathrm{h}}\right)=1.5 \mathrm{~L} / \mathrm{min}$; estimated maximal total portal concentration $\left(\mathrm{I}_{\mathrm{in}, \max }\right)=38.8 \mu \mathrm{M}$; estimated maximal unbound portal concentration $\left(\mathrm{I}_{\mathrm{in}, \mathrm{max}, \mathrm{u}}\right)=17.8 \mu \mathrm{M}$.

$$
R-\text { value }=1+f_{u, p} \times I_{\text {in, } \max } / I C_{50} \quad I_{\text {in, } \max }=I_{\text {max }}+\frac{f_{a} \times f_{g} \times k_{a} \times \text { Dose }}{Q_{h} \times R_{B}}
$$




\begin{tabular}{|c|c|c|c|c|c|c|c|}
\hline Compd & Formula & $\mathrm{RT}$ (min) & $\begin{array}{l}\text { Precursor } \\
\text { Exact } \mathrm{m} / \mathrm{z}\end{array}$ & $\begin{array}{l}\text { Mass Transition for } \\
\text { MS/HRMS } \\
\text { (Precursor>Product) }\end{array}$ & Calibration Range (ng/mL) & $\mathrm{R}^{2}$ & $\begin{array}{r}\text { LOQ } \\
(\mathrm{ng} / \mathrm{mL})\end{array}$ \\
\hline Creatinine & $\mathrm{C}_{4} \mathrm{H}_{7} \mathrm{~N}_{3} \mathrm{O}$ & 1.56 & 114.066 & $114.1>44.0500$ & $20-20000$ & 0.999 & 20 \\
\hline Metformin & $\mathrm{C}_{4} \mathrm{H}_{11} \mathrm{~N}_{5}$ & 2.17 & 130.109 & $130.1>71.0600$ & $0.5-20000$ & 0.997 & 0.5 \\
\hline $\mathrm{N}^{1}$-Methylnicotinamide & $\mathrm{C}_{7} \mathrm{H}_{8} \mathrm{~N}_{2} \mathrm{O}$ & 3.53 & 137.071 & $137.1>94.0649$ & $1-1000$ & 0.999 & 1 \\
\hline Isobutyryl-L-carnitine & $\mathrm{C}_{11} \mathrm{H}_{21} \mathrm{NO}_{4}$ & 4.11 & 232.154 & $232.1>85.0300$ & $0.5-500$ & 0.999 & 0.5 \\
\hline Thiamine & $\mathrm{C}_{12} \mathrm{H}_{17} \mathrm{~N}_{4} \mathrm{OS}$ & 4.64 & 265.115 & $265.1>122.0709$ & $0.5-100$ & 0.999 & 0.5 \\
\hline Carnitine & $\mathrm{C}_{7} \mathrm{H}_{15} \mathrm{NO}_{3}$ & 4.79 & 162.113 & $162.1>85.0280$ & $50-20000$ & 0.998 & 50 \\
\hline D3-creatinine & $\mathrm{C}_{4} \mathrm{H}_{4} \mathrm{D}_{3} \mathrm{~N}_{3} \mathrm{O}$ & 1.56 & 117.084 & $117.1>47.0683$ & & & \\
\hline D6-metformin & $\mathrm{C}_{4} \mathrm{H}_{5} \mathrm{D}_{6} \mathrm{~N}_{5}$ & 2.17 & 136.147 & $136.1>77.0972$ & & & \\
\hline 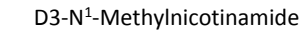 & $\mathrm{C}_{7} \mathrm{H}_{5} \mathrm{D}_{3} \mathrm{~N}_{2} \mathrm{O}$ & 3.53 & 140.089 & $140.1>97.0835$ & & & \\
\hline D3-isobutyryl-L-carnitine & $\mathrm{C}_{11} \mathrm{H}_{18} \mathrm{D}_{3} \mathrm{NO}_{4}$ & 4.11 & 235.173 & $235.1>85.0282$ & & & \\
\hline D3-thiamine & $\mathrm{C}_{12} \mathrm{H}_{14} \mathrm{D}_{3} \mathrm{~N}_{4} \mathrm{OS}$ & 4.64 & 268.128 & $268.1>125.0900$ & & & \\
\hline D3-carnitine & $\mathrm{C}_{7} \mathrm{H}_{12} \mathrm{D}_{3} \mathrm{NO}_{3}$ & 4.79 & 165.131 & $165.1>85.0285$ & & & \\
\hline \multicolumn{8}{|l|}{ Full Scan Experiment or } \\
\hline HILIC-HRMS or TOF-MS & & & $40-540$ & & & & \\
\hline
\end{tabular}

Table S2. Analytical Characteristics of the UHPLC-MS/HRMS Method. 
Table S3: Slope comparison demonstrating parallelism in authentic and surrogate matrices for the endogenous analytes

\begin{tabular}{|c|c|c|c|}
\hline & \multicolumn{2}{|c|}{ Slope } & \%Diff \\
\hline Analyte & Authentic & Surrogate & \\
\hline IBC & 0.0285 & 0.0276 & -3.2 \\
\hline Creatinine & 0.0144 & 0.0151 & 4.7 \\
\hline 1-NMN & 0.0248 & 0.0242 & -2.4 \\
\hline Thiamine & 0.0222 & 0.0234 & 5.3 \\
\hline Carnitine & 0.0546 & 0.0601 & 9.6 \\
\hline
\end{tabular}


Table S4. Intra- and Inter-day Assay Precision of the Endogenous Analytes in Human Control Plasma.

\begin{tabular}{|c|c|c|c|c|}
\hline \multirow{2}{*}{ Sample \# } & \multicolumn{2}{|c|}{ Intraday Precision } & \multicolumn{2}{|c|}{ Inter-day Precision } \\
\hline & Mean $(n=4)$ & CV\% & Mean $(n=3)$ & CV\% \\
\hline \multicolumn{5}{|c|}{ IBC } \\
\hline 1 & 11.91 & 3.9 & 11.49 & 7.9 \\
\hline 2 & 10.88 & 4.1 & 10.07 & 11.0 \\
\hline 3 & 33.54 & 3.8 & 30.90 & 10.7 \\
\hline 4 & 32.22 & 3.2 & 29.78 & 10.6 \\
\hline 5 & 20.96 & 3.2 & 19.55 & 11.3 \\
\hline 6 & 34.67 & 6.8 & 32.37 & 9.4 \\
\hline \multicolumn{5}{|c|}{ 1-NMN } \\
\hline 1 & 19.99 & 14.6 & 19.43 & 11.2 \\
\hline 2 & 8.89 & 5.7 & 8.38 & 8.6 \\
\hline 3 & 27.34 & 3.3 & 23.94 & 14.0 \\
\hline 4 & 16.36 & 3.4 & 14.88 & 10.4 \\
\hline 5 & 10.53 & 3.8 & 9.86 & 7.6 \\
\hline 6 & 14.52 & 3.5 & 13.07 & 11.4 \\
\hline \multicolumn{5}{|c|}{ Carnitine } \\
\hline 1 & 3889.61 & 8.5 & 3838.14 & 6.2 \\
\hline 2 & 4985.67 & 6.8 & 4886.90 & 8.4 \\
\hline 3 & 5235.78 & 5.6 & 5012.19 & 6.5 \\
\hline 4 & 5330.08 & 12.1 & 5231.78 & 8.4 \\
\hline 5 & 4897.30 & 11.8 & 4750.26 & 9.5 \\
\hline 6 & 5606.32 & 2.6 & 5536.69 & 3.2 \\
\hline \multicolumn{5}{|c|}{ Creatinine } \\
\hline 1 & 5816.01 & 9.3 & 5644.17 & 7.1 \\
\hline 2 & 6191.82 & 3.2 & 6025.62 & 3.9 \\
\hline 3 & 8786.61 & 4.8 & 8557.07 & 4.7 \\
\hline 4 & 7541.14 & 6.5 & 7314.45 & 6.7 \\
\hline 5 & 7393.28 & 6.0 & 7298.49 & 4.5 \\
\hline 6 & 8005.73 & 2.0 & 7906.05 & 2.3 \\
\hline \multicolumn{5}{|c|}{ Thiamine } \\
\hline 1 & 2.47 & 11.1 & 2.48 & 14.8 \\
\hline 2 & 1.44 & 12.5 & 1.27 & 19.0 \\
\hline 3 & 1.90 & 3.9 & 1.79 & 11.1 \\
\hline 4 & 1.15 & 6.0 & 1.08 & 12.7 \\
\hline 5 & 0.57 & 8.0 & 0.61 & 16.9 \\
\hline 6 & 1.07 & 16.2 & 1.10 & 13.2 \\
\hline
\end{tabular}


Table S5: Matrix Effect for the Six Analytes in Human Plasma

\begin{tabular}{ccccc} 
Analyte & Spiked Conc. $(\mathrm{ng} / \mathrm{mL})$ & MF of Analyte & MF of IS & IS-Normalized MF \\
IBC & 200 & 1.01 & 0.99 & 1.03 \\
\multirow{2}{*}{$1-\mathrm{NMN}$} & 500 & 1.07 & 1.10 & 0.98 \\
& 200 & 1.37 & 1.32 & 1.04 \\
Thiamine & 500 & 1.67 & 1.75 & 0.97 \\
& 50 & 0.95 & 1.09 & 0.91 \\
Metformin & 100 & 0.96 & 0.92 & 1.08 \\
& 200 & 1.17 & 1.06 & 1.11 \\
Carnitine & 500 & 1.09 & 1.08 & 1.02 \\
Creatinine & 10000 & $1.61^{*}$ & 1.53 & $1.05^{*}$ \\
& 20000 & $1.33^{*}$ & 1.31 & $1.02^{*}$ \\
& 10000 & $1.84^{*}$ & 1.71 & $1.07^{*}$
\end{tabular}

* Analyte response was corrected for endogenous levels of analyte measured in the blank matrix. 
Table S6: Recovery of the Six Analytes in Human Plasma

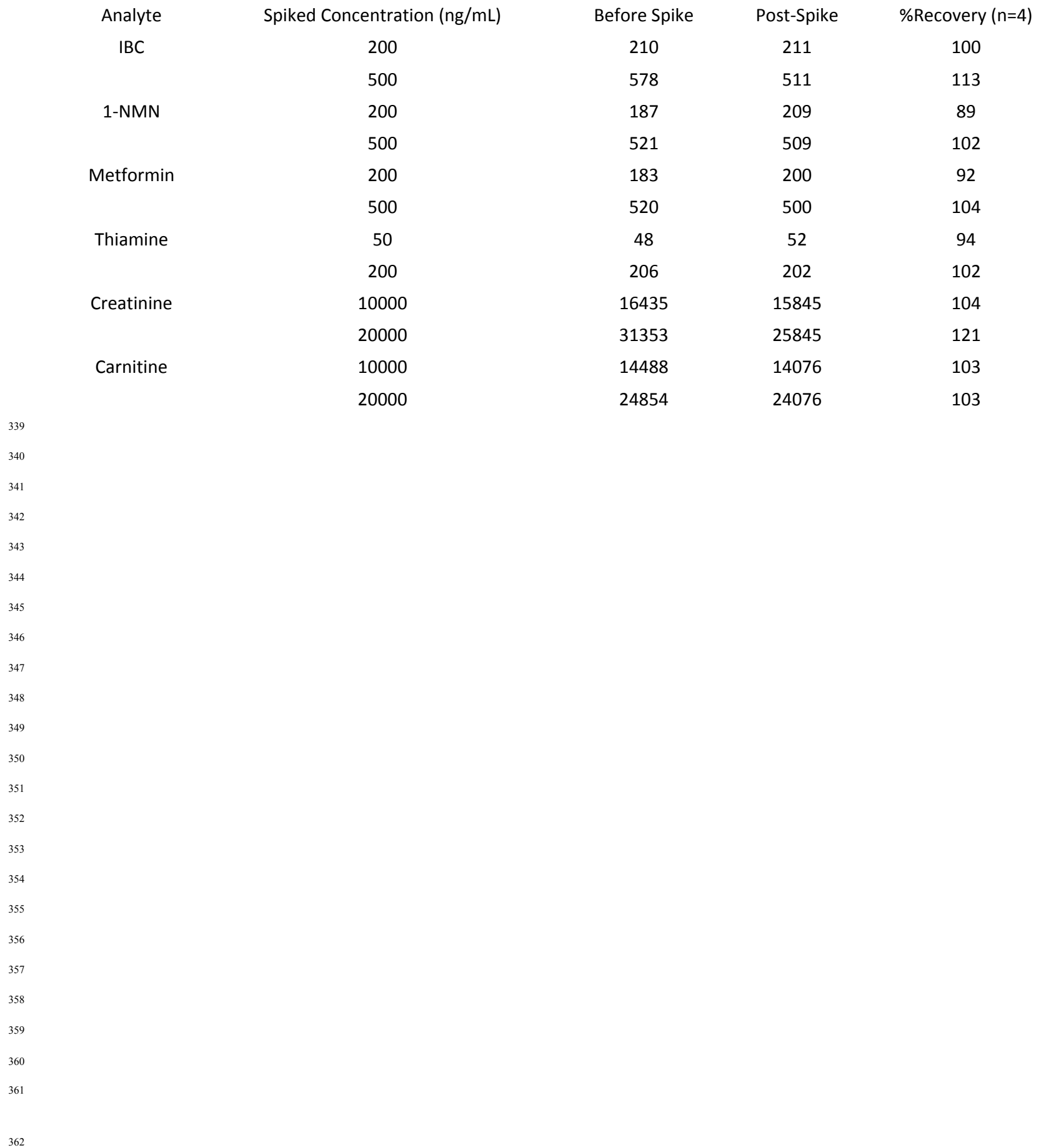

